\title{
Exact Solutions Superimposed with Nonlinear Plane Waves
}

\author{
B. S. Desale ${ }^{1}$ and Vivek Sharma ${ }^{2}$ \\ ${ }^{1}$ Department of Mathematics, University of Mumbai, Mumbai 400 098, India \\ ${ }^{2}$ Department of Mathematics, North Maharashtra University, Jalgaon 425 001, India \\ Correspondence should be addressed to B. S. Desale; bhausaheb.desale@mathematics.mu.ac.in
}

Received 9 February 2016; Accepted 20 April 2016

Academic Editor: Wen-Xiu Ma

Copyright (C) 2016 B. S. Desale and V. Sharma. This is an open access article distributed under the Creative Commons Attribution License, which permits unrestricted use, distribution, and reproduction in any medium, provided the original work is properly cited.

\begin{abstract}
The flow of fluid in atmosphere and ocean is governed by rotating stratified Boussinesq equations. Through the literature, we found that many researchers are trying to find the solutions of rotating stratified Boussinesq equations. In this paper, we have obtained special exact solutions and nonlinear plane waves. Finally, we provide exact solutions to rotating stratified Boussinesq equations with large scale motion superimposed with the nonlinear plane waves. In support of our investigations, we provided two examples: one described the special exact solution and in second example, we have determined the special exact solution superimposed with nonlinear plane wave. Also, we depicted some integral curves that represent the flow of an incompressible fluid particle on the plane $x_{1}+x_{2}=L$ (constant) as the particular case.
\end{abstract}

\section{Introduction}

In certain ranges of scale in the atmosphere and in the ocean, the flow of fluid is controlled by interaction of gravitational force and the rotation of earth with density variation about reference state. The fluid velocity is too slow to involve incompressible effect. Equations which work on these scales are called the rotating stratified Boussinesq equations, and the gravity waves they support are built up in Gill [1]. It may be noted that the Boussinesq approximation in the literature is also referred to as the Oberbeck-Boussinesq approximation, for which the reader is referred to an interesting article of Rajagopal et al. [2]. Majda and Shefter [3] chose certain special solutions of this system of PDEs to demonstrate onset of instability when the Richardson number is less than $1 / 4$. In their study of instability in stratified fluids at large Richardson number they obtained the exact solutions to stratified Boussinesq equations neglecting the effects of rotations and viscosity. Further, in absence of strain field Srinivasan et al. [4] proved that the reduced system of ODEs is completely integrable. For the similar kind of work reader may refer to articles of Maas [5, 6]. In his monograph Majda [7] has obtained the special solution of rotating stratified Boussinesq equations excluding the effects of viscosity and finite rotation. In our earlier work we have included the effect of earth rotation and obtained the nonlinear plane wave solutions of rotating stratified Boussinesq equations.

In this paper, we continue with the special exact solutions of rotating stratified Boussinesq equations on the large scale motions and nonlinear plane waves. Here, we built up the exact solutions of rotating stratified Boussinesq equations superimposed with nonlinear plane waves followed by two examples, out of which one gives special exact solution and second example provides us the superimposition of nonlinear plane wave with special exact solution of rotating stratified Boussinesq equations.

\section{Special Solutions}

The motion of an incompressible flow of fluid in the atmosphere and in the ocean is governed by the following nondimensional rotating stratified Boussinesq equations:

$$
\begin{aligned}
\frac{D \mathbf{v}}{D t}+\frac{1}{R_{0}} \mathbf{u} & =-\bar{P} \nabla p-\Gamma \widehat{\mathbf{e}_{3}}, \\
\operatorname{div} \mathbf{v} & =0, \\
\frac{D \widetilde{\rho}}{D t} & =\frac{D \rho}{D t}+\left(\frac{d \bar{\rho}}{d x_{3}}\right) v^{3}=0 .
\end{aligned}
$$


Here, we have that $\mathbf{v}=\left(v^{1}, v^{2}, v^{3}\right), \mathbf{u}=\left(u^{1}, u^{2}, u^{3}\right)=\widehat{\mathbf{e}_{3}} \times \mathbf{v}, \Gamma$ is the nondimensional number, $R_{0}$ is the Rossby number, and $\bar{P}$ is the Euler number. Nondimensional density function is

$$
\widetilde{\rho}(\mathbf{x}, t)=\rho_{b}+\bar{\rho}\left(x_{3}\right)+\rho(\mathbf{x}, t) .
$$

We make the usual assumption valid for local consideration that $d \bar{\rho} / d x_{3}$ is constant and it is the integrated part of $\rho_{b}$. Henceforth, we have $\tilde{\rho}(\mathbf{x}, t)=\rho_{b}+\rho(\mathbf{x}, t)$. For more details one may refer to our earlier article [8]. The more elaborative discussion about the nondimensional analysis of rotating stratified Boussinesq equations is found in the monograph of Majda [7].

Now, we present the special solutions of (1) in large scale motion of fluid. For local behavior of incompressible fluid, we have Taylor's series expansion for smooth velocity field and density function about some point $\mathbf{x}_{0}$ :

$$
\begin{aligned}
& \mathbf{v}(\mathbf{x}, t)=\mathbf{v}\left(\mathbf{x}_{0}, t\right)+\left.\nabla \mathbf{v}\right|_{\left(\mathbf{x}_{0}, t\right)}\left(\mathbf{x}-\mathbf{x}_{0}\right)+O\left(\left|\mathbf{x}-\mathbf{x}_{0}\right|^{2}\right), \\
& \tilde{\rho}(\mathbf{x}, t)=\rho_{b}+\left.\nabla \widetilde{\rho}\right|_{\left(\mathbf{x}_{0}, t\right)}\left(\mathbf{x}-\mathbf{x}_{0}\right)+O\left(\left|\mathbf{x}-\mathbf{x}_{0}\right|^{2}\right),
\end{aligned}
$$

where $\nabla \mathbf{v}$ is a $3 \times 3$ matrix whose $(i, j)$ th entry is $\partial v^{i} / \partial x_{j}$, where $i=1,2,3$ and $j=1,2,3$. Equation (4) is the decomposition of the matrix $\nabla \mathbf{v}$ as a sum of symmetric and skew symmetric matrices and this kind of decomposition is unique:

$$
\begin{aligned}
\left.\nabla \mathbf{v}\right|_{\left(\mathbf{x}_{0}, t\right)} & =\left(\frac{\nabla \mathbf{v}+(\boldsymbol{\nabla} \mathbf{v})^{T}}{2}\right)+\left(\frac{\nabla \mathbf{v}-(\nabla \mathbf{v})^{T}}{2}\right) \\
& =\mathscr{D}\left(\mathbf{x}_{0}, t\right)+\Omega\left(\mathbf{x}_{0}, t\right)=V\left(\mathbf{x}_{0}, t\right),
\end{aligned}
$$

where $\mathscr{D}$ is the symmetric part of $\nabla \mathbf{v}$ and is called deformation matrix; it has the property that the trace of matrix $\mathscr{D}$ is equal to the divergence of vector field $\mathbf{v}$, whereas $\Omega$ is a skew symmetric part of matrix $\nabla \mathbf{v}$ and satisfies the following equation:

$$
\Omega \mathbf{h}=\frac{1}{2} \mathbf{w} \times \mathbf{h},
$$

for any vector $\mathbf{h} \in \mathbb{R}^{3}$. The vector $\mathbf{w}$ is vorticity vector; that is, $\mathbf{w}=\boldsymbol{\nabla} \times \mathbf{v}=\left(w_{1}, w_{2}, w_{3}\right)$. Hence, from (4) we get

$$
\left.\nabla \mathbf{v}\right|_{\left(\mathbf{x}_{0}, t\right)} \mathbf{h}=\mathscr{D}\left(\mathbf{x}_{0}, t\right) \mathbf{h}+\frac{1}{2} \mathbf{w}\left(\mathbf{x}_{0}, t\right) \times \mathbf{h} .
$$

The decomposition of a vector $\mathbf{v}$ as in (3) by mean of (4) has a simple physical interpretation; namely, every incompressible velocity field is a sum of translation, stretching, and rotation. We may deprive the translation part by a Galilean transformation. We assume that $\mathbf{v}\left(\mathbf{x}_{0}, t\right)=0$. A gradient of $\mathbf{u}$ is a matrix $\left(u_{x_{k}}^{i}\right)$ which can be uniquely expressed as $\left(u_{x_{k}}^{i}\right)=(S+Q)$, where the symmetric matrix $S$ and skew symmetric matrix $Q$ are as given below:

$$
S=\frac{1}{2}\left(\begin{array}{ccc}
-2 \frac{\partial v^{1}}{\partial x_{1}} & \frac{\partial v^{1}}{\partial x_{1}}-\frac{\partial v^{2}}{\partial x_{2}} & -\frac{\partial v^{2}}{\partial x_{3}} \\
\frac{\partial v^{1}}{\partial x_{1}}-\frac{\partial v^{2}}{\partial x_{2}} & 2 \frac{\partial v^{1}}{\partial x_{2}} & \frac{\partial v^{1}}{\partial x_{3}} \\
-\frac{\partial v^{2}}{\partial x_{3}} & \frac{\partial v^{1}}{\partial x_{3}} & 0
\end{array}\right),
$$

For any $\mathbf{h} \in \mathbb{R}^{3}$, a skew symmetric matrix $Q$ satisfies the following equation:

$$
Q \mathbf{h}=-\frac{1}{2} \frac{\partial \mathbf{v}}{\partial x_{3}} \times \mathbf{h}
$$

Through the literature we see that at the large scale motion (1) admit the special solutions, which are given in the form of Theorem 1.

Theorem 1. The rotating stratified Boussinesq equations (1) admit the special solutions of the form

$$
\begin{aligned}
\mathbf{v}(\mathbf{x}, t) & =\mathscr{D}(t) \mathbf{x}+\frac{1}{2} \mathbf{w}(t) \times \mathbf{x}, \\
\widetilde{\rho} & =\rho_{b}+\mathbf{b}(t) \cdot \mathbf{x}, \\
\bar{P} p & =\frac{1}{2} \widehat{P}(t) \mathbf{x} \cdot \mathbf{x},
\end{aligned}
$$

where $\bar{P}$ is nondimensional number as defined in (1) and $\mathscr{D}(t)$ is symmetric matrix with zero trace; when $\mathbf{w}(t)=\nabla \times \mathbf{v}$ and $\mathbf{b}(t)=\nabla \widetilde{\rho}$ satisfy the ODEs,

$$
\begin{aligned}
\frac{d \mathbf{w}}{d t}= & \mathscr{D}(t)\left[\mathbf{w}(t)+\frac{1}{R_{0}} \widehat{\mathbf{e}_{3}}\right]+\Gamma \widehat{\mathbf{e}_{3}} \times \mathbf{b}(t)-\frac{1}{2 R_{0}} \widehat{\mathbf{e}_{3}} \\
& \times \mathbf{w}(t), \\
\frac{d \mathbf{b}}{d t}= & -\mathscr{D}(t) \mathbf{b}(t)+\frac{1}{2} \mathbf{w}(t) \times \mathbf{b}(t),
\end{aligned}
$$

and matrix $\widehat{P}(t)$ is given by

$$
-\widehat{P}=\frac{d \mathscr{D}}{d t}+\mathscr{D}^{2}+\Omega^{2}+\frac{1}{R_{0}} S+\frac{\Gamma}{2}\left(\widehat{\mathbf{e}_{3}} \mathbf{b}^{T}+\mathbf{b}{\widehat{\mathbf{e}_{3}}}^{T}\right),
$$

where the matrix $\Omega$ is as defined in (4) through the linear map given by (5) and the matrix $S$ is given by (7).

In Section 3, we present the nonlinear plane wave solutions of rotating stratified Boussinesq equations. 


\section{Nonlinear Plane Waves}

In the development of exact solutions of (1) in the form of nonlinear plane waves, the following trivial Lemma 2 is a useful step.

Lemma 2. For $\mathbf{v}$ of the form $\mathbf{v}=\mathbf{A}(t) F(\boldsymbol{\alpha}(t) \cdot \mathbf{x}), \operatorname{div} \mathbf{v}=0$ implies

(i) $\mathbf{A}(t) \cdot \boldsymbol{\alpha}(t)=0$;

(ii) $\mathbf{v} \cdot \nabla W(\boldsymbol{\alpha}(t) \cdot \mathbf{x})=0$,

for arbitrary $W$, where $\mathbf{A}(t)=\left(A_{1}(t), A_{2}(t), A_{3}(t)\right)$ and $\boldsymbol{\alpha}(t)=$ $\left(\alpha_{1}(t), \alpha_{2}(t), \alpha_{3}(t)\right)$.

For the proof of this lemma one may refer to Majda [7, pp. 20]. Theorem 3 describes the nonlinear plane wave solutions of (1).

Theorem 3. The rotating stratified Boussinesq equations (1) admit the exact solutions in the form of nonlinear plane waves as follows:

$$
\begin{aligned}
& \mathbf{v}=\mathbf{A}(t) F(\boldsymbol{\alpha}(t) \cdot \mathbf{x}), \\
& \rho=B(t) F(\boldsymbol{\alpha}(t) \cdot \mathbf{x}), \\
& p=P(t) G(\boldsymbol{\alpha}(t) \cdot \mathbf{x}),
\end{aligned}
$$

where $F$ and $G$ are arbitrary functions of $\boldsymbol{\alpha}(t) \cdot \mathbf{x}$ with the condition $G^{\prime}(s)=F(s)$ provided that $\boldsymbol{\alpha}(t), \mathbf{A}(t), B(t)$, and $P(t)$ satisfy the following equations:

$$
\frac{d \boldsymbol{\alpha}(t)}{d t}=0
$$

$\mathbf{A}(t) \cdot \boldsymbol{\alpha}(t)=0$,

$P(t)$

$$
=-\frac{1}{R_{0} \bar{P}}\left(\frac{\boldsymbol{\alpha}(t) \cdot\left(\widehat{\mathbf{e}_{3}} \times \mathbf{A}(t)\right)}{|\boldsymbol{\alpha}(t)|^{2}}\right)-\frac{\Gamma \alpha_{3}(t)}{\bar{P}|\boldsymbol{\alpha}(t)|^{2}} B(t),
$$

$\frac{d \mathbf{A}(t)}{d t}$

$$
\begin{aligned}
= & \frac{-1}{R_{0}}\left(\widehat{\mathbf{e}_{3}} \times \mathbf{A}(t)\right) \\
& +\left[\frac{\boldsymbol{\alpha}(t) \cdot\left(\widehat{\mathbf{e}_{3}} \times \mathbf{A}(t)\right)}{R_{0}|\boldsymbol{\alpha}(t)|^{2}}+\frac{\Gamma \alpha_{3}(t)}{|\boldsymbol{\alpha}(t)|^{2}} B(t)\right] \boldsymbol{\alpha}(t) \\
& -\Gamma B(t) \widehat{\mathbf{e}_{3}},
\end{aligned}
$$$$
\frac{d B(t)}{d t}+\frac{d \bar{\rho}}{d x_{3}} A_{3}(t)=0 \text {. }
$$

\section{Superimposed Nonlinear Plane Wave Solutions}

In Section 2, we obtained exact solutions of rotating stratified Boussinesq equations for the large scale motion of fluids, while in Section 3, we have determined the nonlinear plane wave solutions. In this section we aim at presenting the special exact solutions of (1) superimposed with nonlinear plane waves. We have obtained such solutions and presented them in the form of Theorem 6. Prior to determining the superimposed solutions, we have some key elements in the form of lemmas. The proof of these lemmas is essential to develop these superimposed solutions.

Lemma 4. Let $V$ be as defined in (4) and let $\boldsymbol{\alpha}, \mathbf{A}$ be any two vectors in $\mathbb{R}^{3}$; then one has

$$
V^{T} \boldsymbol{\alpha} \cdot \mathbf{A}=V \mathbf{A} \cdot \boldsymbol{\alpha}
$$

Proof. We have $V=\mathscr{D}+\Omega$ as defined in (4), so that $V^{T}=$ $(\mathscr{D}+\Omega)^{T}=\mathscr{D}-\Omega$. Hence,

$$
\begin{aligned}
V^{T} \boldsymbol{\alpha} \cdot \mathbf{A} & =(\mathscr{D}+\Omega)^{T} \boldsymbol{\alpha} \cdot \mathbf{A}=[(\mathscr{D}-\Omega) \boldsymbol{\alpha}] \cdot \mathbf{A} \\
& =\left[\mathscr{D} \boldsymbol{\alpha}-\frac{1}{2} \mathbf{w} \times \boldsymbol{\alpha}\right] \cdot \mathbf{A} \\
& =\left[\mathscr{D A}+\frac{1}{2} \mathbf{w} \times \mathbf{A}\right] \cdot \boldsymbol{\alpha}=[(\mathscr{D}+\Omega) \mathbf{A}] \cdot \boldsymbol{\alpha} \\
& =V \mathbf{A} \cdot \boldsymbol{\alpha} .
\end{aligned}
$$

Lemma 5. Let $V$ be as defined in (4) and let $S$ and $Q$ be the symmetric and skew symmetric matrices, respectively, as defined in (7); then one has

$$
\frac{1}{R_{0}} \widehat{\mathbf{e}_{3}} \times(V \mathbf{x})=\frac{1}{R_{0}}(S+Q) \mathbf{x}
$$

Proof. The term $V$ as defined in (4) is nothing but $\nabla \mathbf{v}$; that is,

$$
V=\left(\begin{array}{lll}
\frac{\partial v^{1}}{\partial x_{1}} & \frac{\partial v^{1}}{\partial x_{2}} & \frac{\partial v^{1}}{\partial x_{3}} \\
\frac{\partial v^{2}}{\partial x_{1}} & \frac{\partial v^{2}}{\partial x_{2}} & \frac{\partial v^{2}}{\partial x_{3}} \\
\frac{\partial v^{3}}{\partial x_{1}} & \frac{\partial v^{3}}{\partial x_{2}} & \frac{\partial v^{3}}{\partial x_{3}}
\end{array}\right)
$$

Hence, we have

$$
V \mathbf{x}=\left(\begin{array}{c}
x_{1} \frac{\partial v^{1}}{\partial x_{1}}+x_{2} \frac{\partial v^{1}}{\partial x_{2}}+x_{3} \frac{\partial v^{1}}{\partial x_{3}} \\
x_{1} \frac{\partial v^{2}}{\partial x_{1}}+x_{2} \frac{\partial v^{2}}{\partial x_{2}}+x_{3} \frac{\partial v^{2}}{\partial x_{3}} \\
x_{1} \frac{\partial v^{3}}{\partial x_{1}}+x_{2} \frac{\partial v^{3}}{\partial x_{2}}+x_{3} \frac{\partial v^{3}}{\partial x_{3}}
\end{array}\right)
$$


Furthermore, we calculate

$$
\begin{aligned}
& \frac{1}{R_{0}} \widehat{\mathbf{e}_{3}} \times(V \mathbf{x}) \\
& \quad=\frac{1}{R_{0}}\left(\begin{array}{c}
-x_{1} \frac{\partial v^{2}}{\partial x_{1}}-x_{2} \frac{\partial v^{2}}{\partial x_{2}}-x_{3} \frac{\partial v^{2}}{\partial x_{3}} \\
x_{1} \frac{\partial v^{1}}{\partial x_{1}}+x_{2} \frac{\partial v^{1}}{\partial x_{2}}+x_{3} \frac{\partial v^{1}}{\partial x_{3}} \\
0
\end{array}\right) .
\end{aligned}
$$

In the similar fashion, we calculate the vector $\left(1 / R_{0}\right)(S+Q) \mathbf{x}$ as follows:

$$
\begin{aligned}
& \frac{1}{R_{0}}(S+Q) \mathbf{x} \\
& =\frac{1}{R_{0}}\left(\begin{array}{c}
-x_{1} \frac{\partial v^{2}}{\partial x_{1}}-x_{2} \frac{\partial v^{2}}{\partial x_{2}}-x_{3} \frac{\partial v^{2}}{\partial x_{3}} \\
x_{1} \frac{\partial v^{1}}{\partial x_{1}}+x_{2} \frac{\partial v^{1}}{\partial x_{2}}+x_{3} \frac{\partial v^{1}}{\partial x_{3}} \\
0
\end{array}\right)
\end{aligned}
$$

and (19) and (20) lead to the conclusion of the lemma.

Now, we present the superimposed solutions of rotating stratified Boussinesq equations (1) which are the superposition of exact solutions and nonlinear plane wave solutions. We describe these solutions in the form of Theorem 6 .

Theorem 6. There are special exact solutions to the rotating stratified Boussinesq equations (1) in the form of

$$
\begin{aligned}
\mathbf{v}(\mathbf{x}, t) & =\mathscr{D}(t) \mathbf{x}+\frac{1}{2} \mathbf{w}(t) \times \mathbf{x}+\mathbf{A}(t) F(\boldsymbol{\alpha}(t) \cdot \mathbf{x}) \\
\widetilde{\rho} & =\rho_{b}+\mathbf{b}(t) \cdot \mathbf{x}+B(t) F(\boldsymbol{\alpha}(t) \cdot \mathbf{x}) \\
& =\rho_{b}+\rho(\mathbf{x}, t) \\
p & =\frac{1}{2 \bar{P}} \widehat{P}(t) \mathbf{x} \cdot \mathbf{x}+P(t) G(\boldsymbol{\alpha}(t) \cdot \mathbf{x})
\end{aligned}
$$

where $G^{\prime}(s)=F(s)$ and $\mathscr{D}$ is an arbitrary $3 \times 3$, traceless, symmetric matrix as defined in (4) provided that $\mathbf{w}(t)$ and $\mathbf{b}(t)$ satisfy the ODEs (10) along with the wave phase and amplitudes satisfy the following ODEs:

$$
\begin{aligned}
& \frac{d \boldsymbol{\alpha}(t)}{d t}=-V^{T}(t) \boldsymbol{\alpha}(t), \\
& \frac{d \mathbf{A}(t)}{d t} \\
& =-V(t) \mathbf{A}(t)+\boldsymbol{\alpha}(t) \frac{2\left(V^{T}(t) \boldsymbol{\alpha}(t) \cdot \mathbf{A}(t)\right)}{|\boldsymbol{\alpha}(t)|^{2}} \\
& \quad-\frac{1}{R_{0}}\left(\widehat{\mathbf{e}_{3}} \times \mathbf{A}(t)\right)
\end{aligned}
$$

$$
\begin{aligned}
+ & {\left[\frac{\boldsymbol{\alpha}(t) \cdot\left(\widehat{\mathbf{e}_{3}} \times \mathbf{A}(t)\right)}{R_{0}|\boldsymbol{\alpha}(t)|^{2}}+\frac{\Gamma \alpha_{3}(t)}{|\boldsymbol{\alpha}(t)|^{2}} B(t)\right] \boldsymbol{\alpha}(t) } \\
- & \Gamma B(t) \widehat{\mathbf{e}_{3}}, \\
\frac{d B(t)}{d t} & =-\mathbf{A}(t) \cdot \mathbf{b}(t),
\end{aligned}
$$

where $V(t)=\mathscr{D}(t)+\Omega(t)$ as defined in (4). The initial conditions are arbitrary, except that one requires

$$
\left.\boldsymbol{\alpha}(t) \cdot \mathbf{A}(t)\right|_{t=0}=0
$$

The matrix $\widehat{P}(t)$ in pressure term satisfies the differential equation (11) and scalar function $P(t)$ in pressure term is calculated as

$$
\begin{aligned}
-P(t)= & \frac{2\left(V^{T}(t) \boldsymbol{\alpha}(t) \cdot \mathbf{A}(t)\right)}{\bar{P}|\boldsymbol{\alpha}(t)|^{2}} \\
& +\frac{1}{R_{0} \bar{P}}\left(\frac{\boldsymbol{\alpha}(t) \cdot\left(\widehat{\boldsymbol{e}_{3}} \times \mathbf{A}(t)\right)}{|\boldsymbol{\alpha}(t)|^{2}}\right) \\
& +\frac{\Gamma \alpha_{3}(t)}{\bar{P}|\boldsymbol{\alpha}(t)|^{2}} B(t)
\end{aligned}
$$

Proof. We proceed towards the proof of the theorem. In the beginning, we consider that $\mathbf{v}=\mathbf{v}(\mathbf{x}, t), V=V(t), \mathbf{A}=\mathbf{A}(t)$, $\boldsymbol{\alpha}=\boldsymbol{\alpha}(t), B=B(t), P=P(t), \widehat{P}=\widehat{P}(t), F(\boldsymbol{\alpha}(t) \cdot \mathbf{x})=F(\boldsymbol{\alpha} \cdot \mathbf{x})=$ $F$, and $G(\boldsymbol{\alpha}(t) \cdot \mathbf{x})=G(\boldsymbol{\alpha} \cdot \mathbf{x})=G$ for handy use of calculations throughout the proof.

To prove this theorem we have to verify that the velocity $\mathbf{v}$, density function $\widetilde{\rho}$, and pressure $p$ which are given by (21) satisfy (1).

Let us consider the derivative of $\mathbf{A} \cdot \boldsymbol{\alpha}$ which is given by

$$
\frac{d}{d t}(\mathbf{A} \cdot \boldsymbol{\alpha})=\frac{d \mathbf{A}}{d t} \cdot \boldsymbol{\alpha}+\mathbf{A} \cdot \frac{d \boldsymbol{\alpha}}{d t}
$$

Substituting $d \boldsymbol{\alpha} / d t$ and $d \mathbf{A} / d t$ from (22) into the right hand side of (25) and then simplifying the right hand side with the use of Lemma 4 , we get $(d / d t)(\mathbf{A} \cdot \boldsymbol{\alpha})=0$. Together with initial condition (23), we conclude that

$$
\mathbf{A} \cdot \boldsymbol{\alpha}=0
$$

for all times. Now, we proceed towards the requirements for an incompressible fluid; we have $V=\mathscr{D}+\Omega$ so that $\mathbf{v}=V \mathbf{x}+$ FA. Therefore, $\operatorname{div} \mathbf{v}=$ trace $\mathscr{D}+(\mathbf{A} \cdot \boldsymbol{\alpha}) F^{\prime} . F$ is arbitrary and $\mathscr{D}$ is symmetric traceless strain matrix so that trace $\mathscr{D}=0$, and also we have $\mathbf{A} \cdot \boldsymbol{\alpha}=0$ for all times. Hence $\mathbf{v}$ satisfy the equation $\operatorname{div} \mathbf{v}=0$.

Our next aim is to verify the momentum equation. We now compute the advective derivative:

$$
\frac{\partial \mathbf{v}}{\partial t}=\frac{d V}{d t} \mathbf{x}+F \frac{d \mathbf{A}}{d t}+F^{\prime}\left(\frac{d \boldsymbol{\alpha}}{d t} \cdot \mathbf{x}\right) \mathbf{A} .
$$


The advection caused by velocity is calculated as

$$
\begin{aligned}
(\mathbf{v} \cdot \nabla) \mathbf{v} & =[(V \mathbf{x}+F \mathbf{A}) \cdot \nabla][V \mathbf{x}+F \mathbf{A}] \\
& =V^{2} \mathbf{x}+F V \mathbf{A}+(V \mathbf{x} \cdot \boldsymbol{\alpha}) F^{\prime} \mathbf{A} \\
& =V^{2} \mathbf{x}+F V \mathbf{A}+\left(V^{T} \boldsymbol{\alpha} \cdot \mathbf{x}\right) F^{\prime} \mathbf{A}
\end{aligned}
$$

because of Lemma 4.

Adding (27) and (28), we obtain the advective derivative of $\mathbf{v}$ as

$$
\begin{aligned}
\frac{D \mathbf{v}}{D t}= & \left(\frac{d V}{d t}+V^{2}\right) \mathbf{x}+F\left(\frac{d \mathbf{A}}{d t}+V \mathbf{A}\right) \\
& +F^{\prime}\left(\left[\frac{d \boldsymbol{\alpha}}{d t}+V^{T} \boldsymbol{\alpha}\right] \cdot \mathbf{x}\right) \mathbf{A} .
\end{aligned}
$$

Now, we calculate the rotation term of momentum equation:

$$
\begin{aligned}
\frac{1}{R_{0}} \mathbf{u} & =\frac{1}{R_{0}} \widehat{\mathbf{e}_{3}} \times \mathbf{v}=\frac{1}{R_{0}} \widehat{\mathbf{e}_{3}} \times[V \mathbf{x}+F \mathbf{A}] \\
& =\frac{1}{R_{0}} \widehat{\mathbf{e}_{3}} \times(V \mathbf{x})+\frac{1}{R_{0}} F\left(\widehat{\mathbf{e}_{3}} \times \mathbf{A}\right) \\
& =\frac{1}{R_{0}}(S+Q) \mathbf{x}+\frac{1}{R_{0}} F\left(\widehat{\mathbf{e}_{3}} \times \mathbf{A}\right)
\end{aligned}
$$

because of Lemma 5.

With easy calculations, we see that the term due to pressure in momentum equation is expressed as

$$
\bar{P} \nabla p=\widehat{P} \mathbf{x}+\bar{P} P F \boldsymbol{\alpha} .
$$

$\rho=(\mathbf{b} \cdot \mathbf{x})+B F$ implies that

$$
\Gamma \rho \widehat{\mathbf{e}_{3}}=\Gamma(\mathbf{b} \cdot \mathbf{x}) \widehat{\mathbf{e}_{3}}+\Gamma B F \widehat{\mathbf{e}_{3}}=\Gamma\left(\widehat{\mathbf{e}_{3}} \mathbf{b}^{T}\right) \mathbf{x}+\Gamma B F \widehat{\mathbf{e}_{3}} .
$$

Taking the addition of (29), (30), (31), and (32), we find that

$$
\begin{aligned}
\frac{D \mathbf{v}}{D t}+ & \frac{1}{R_{0}} \mathbf{u}+\bar{P} \nabla p+\Gamma \rho \widehat{\mathbf{e}_{3}} \\
= & {\left[\frac{d V}{d t}+V^{2}+\widehat{P}+\frac{1}{R_{0}}(S+Q)+\Gamma\left(\widehat{\mathbf{e}_{3}} \mathbf{b}^{T}\right)\right] \mathbf{x} } \\
& +F\left[\frac{d \mathbf{A}}{d t}+V \mathbf{A}+\frac{1}{R_{0}}\left(\widehat{\mathbf{e}_{3}} \times \mathbf{A}\right)+\bar{P} P \boldsymbol{\alpha}+\Gamma B \widehat{\mathbf{e}_{3}}\right] \\
& +F^{\prime}\left[\left(\frac{d \boldsymbol{\alpha}}{d t}+V^{T} \boldsymbol{\alpha}\right) \cdot \mathbf{x}\right] \mathbf{A} .
\end{aligned}
$$

Since $V=(\mathscr{D}+\Omega)$, it is easy to verify that $V$ satisfies the following equation:

$$
\frac{d V}{d t}+V^{2}+\widehat{P}+\Gamma \widehat{\mathbf{e}_{3}} \mathbf{b}^{T}+\frac{1}{R_{0}}(S+Q)=0 .
$$

Therefore, the first term of right hand side of (33) vanishes. Furthermore, $F$ is arbitrary function of $\boldsymbol{\alpha} \cdot \mathbf{x}, \mathbf{A}$ satisfy the second equation of (22), and substituting the value of $P$ from (24), we verify that the second term in right hand side of (33) becomes zero. Also, we see that third term in right hand side of same equation vanishes because $\boldsymbol{\alpha}$ satisfy the first equation of (22). All these terms together confirm that the right hand side of (33) is identically equal to zero. That is, $D \mathbf{v} / D t+\left(1 / R_{0}\right) \mathbf{u}+\bar{P} \nabla p+\Gamma \rho \widehat{\mathbf{e}_{3}}=0$. Hence, we verified that the velocity $\mathbf{v}$, pressure $p$, and density function $\widetilde{\rho}$ given by (21) together with (22) and (24) satisfy the first equation of (1).

Now, we are at the last step of the proof; here we have to verify the density function $\widetilde{\rho}$ given by (21) which satisfy the density momentum equation of (1), so that we consider

$$
\begin{aligned}
\frac{D \tilde{\rho}}{D t}= & \frac{\partial \widetilde{\rho}}{\partial t}+(\mathbf{v} \cdot \nabla) \tilde{\rho} \\
= & \frac{\partial}{\partial t}\left[\rho_{b}+\mathbf{b} \cdot \mathbf{x}+B F\right] \\
& +[(V \mathbf{x}+F \mathbf{A}) \cdot \nabla]\left[\rho_{b}+\mathbf{b} \cdot \mathbf{x}+B F\right] \\
= & \frac{d \mathbf{b}}{d t} \cdot \mathbf{x}+F \frac{d B}{d t}+B F^{\prime} \frac{d \boldsymbol{\alpha}}{d t} \cdot \mathbf{x}+V \mathbf{x} \cdot \mathbf{b}+F \mathbf{A} \cdot \mathbf{b} \\
& +B F^{\prime} V \mathbf{x} \cdot \boldsymbol{\alpha}+B F F^{\prime} \mathbf{A} \cdot \boldsymbol{\alpha} .
\end{aligned}
$$

The term $B F F^{\prime} \mathbf{A} \cdot \boldsymbol{\alpha}$ of (35) is zero because of (26). Also, we can use Lemma 4 in the above equations and then finally we can obtain the simplified form of (35) as given below:

$$
\begin{aligned}
\frac{D \widetilde{\rho}}{D t}= & {\left[\frac{d \mathbf{b}}{d t}+V^{T} \mathbf{b}\right] \cdot \mathbf{x}+F\left[\frac{d B}{d t}+\mathbf{A} \cdot \mathbf{b}\right] } \\
& +B F^{\prime}\left[\frac{d \boldsymbol{\alpha}}{d t}+V^{T} \boldsymbol{\alpha}\right] \cdot \mathbf{x} .
\end{aligned}
$$

Here, we notice that the second and third terms in the right hand side of (36) are equal to zero because of first and last equations of (22). To see the first term in right hand side of the above equation, we have $V=\mathscr{D}+\Omega$ as a sum of symmetric and skew symmetric matrices, respectively, so that $V^{T}=\mathscr{D}-$ $\Omega$ and $\mathbf{b}$ satisfies (10). Therefore, we have

$$
\frac{d \mathbf{b}}{d t}=-\mathscr{D} \mathbf{b}+\frac{1}{2} \mathbf{w} \times \mathbf{b}=(-\mathscr{D}+\Omega) \mathbf{b}=-V^{T} \mathbf{b} .
$$

This implies that $d \mathbf{b} / d t+V^{T} \mathbf{b}=0$. Thus, we have that the first term in right hand side of (36) is also zero. Hence, $\widetilde{\rho}$ given by (21) satisfy the density momentum equation $D \widetilde{\rho} / D t=0$. Thus we complete the proof of theorem.

\section{Examples}

In this section, we provide two examples that concerns Theorems 3 and 6. Let us start with Example 1.

Example 1. Let us consider vectors $\mathbf{A}=\left(0,0, A_{3}(t)\right)^{T}$ and $\boldsymbol{\alpha}(t)=(1,1,0)^{T}$. Now we define $F(\boldsymbol{\alpha} \cdot \mathbf{x})=F\left(x_{1}+x_{2}\right)=$ $\sin \left(x_{1}+x_{2}\right)$ and $G(\boldsymbol{\alpha} \cdot \mathbf{x})=G\left(x_{1}+x_{2}\right)=-\cos \left(x_{1}+x_{2}\right)$. A scalar function $B(t)$ is now defined as

$$
B(t)=c_{1} \sin (N t)+c_{2} \cos (N t)
$$


and $A_{3}(t)$ is defined as

$$
A_{3}(t)=\Gamma^{1 / 2}\left[c_{1} \cos (N t)-c_{2} \sin (N t)\right]
$$

where $c_{1}, c_{2}$ are arbitrary constants and $N$ is the BruntVäisälä frequency, which has the relation $N=\Gamma^{1 / 2}$ with consideration of $\bar{\rho}=-x_{3}$ for stable stratification. Then, we see that

$$
\begin{aligned}
\mathbf{v}(\mathbf{x}, t) & =\mathbf{A}(t) \sin \left(x_{1}+x_{2}\right), \\
\rho(\mathbf{x}, t) & =B(t) \sin \left(x_{1}+x_{2}\right), \\
\widetilde{\rho}(\mathbf{x}, t) & =\bar{\rho}\left(x_{3}\right)+\rho(\mathbf{x}, t), \\
p & =0 .
\end{aligned}
$$

The velocity, density, and pressure given by (40) represent the nonlinear plane wave solution of (1) as suggested by Theorem 3.

Example 2. Let us consider vectors $\mathbf{A}=\left(0,0, A_{3}(t)\right)^{T}, \boldsymbol{\alpha}(t)=$ $(-1,1,0)^{T}$, and $\mathbf{b}=(0,0,-1)^{T}$. Now we define $F(\boldsymbol{\alpha} \cdot \mathbf{x})=$ $F\left(-x_{1}+x_{2}\right)=\sin \left(-x_{1}+x_{2}\right)$ and $G(\boldsymbol{\alpha} \cdot \mathbf{x})=G\left(-x_{1}+x_{2}\right)=$ $-\cos \left(-x_{1}+x_{2}\right)$. A scalar function $B(t)$ is now defined as

$$
B(t)=c_{1} \sin (N t)+c_{2} \cos (N t)
$$

and $A_{3}(t)$ is defined as

$$
A_{3}(t)=\Gamma^{1 / 2}\left[c_{1} \cos (N t)-c_{2} \sin (N t)\right]
$$

where $c_{1}, c_{2}$ are arbitrary constants and $N$ is the BruntVäisälä frequency, which has the relation $N=\Gamma^{1 / 2}$ with consideration of $\bar{\rho}=-x_{3}$ for stable stratification. Consider the matrices $\mathscr{D}, \Omega$, and $\widehat{P}(t)$ as given below:

$$
\begin{aligned}
\mathscr{D} & =\left(\begin{array}{ccc}
\lambda & 0 & 0 \\
0 & -\lambda & 0 \\
0 & 0 & 0
\end{array}\right), \\
\Omega & =\left(\begin{array}{ccc}
0 & -\lambda & 0 \\
\lambda & 0 & 0 \\
0 & 0 & 0
\end{array}\right), \\
\widehat{P}(t) & =\left(\begin{array}{ccc}
\frac{\lambda}{R_{0}} & -\frac{\lambda}{R_{0}} & 0 \\
-\frac{\lambda}{R_{0}} & \frac{\lambda}{R_{0}} & 0 \\
0 & 0 & -\Gamma
\end{array}\right),
\end{aligned}
$$

where $\lambda$ is a nonzero scalar. With these assumptions we calculate the scalar function $P(t)$ according to formula (24) and this is equal to zero. Then the following is the superimposed solution of (1) suggested by Theorem 6:

$$
\begin{aligned}
& \mathbf{v}(\mathbf{x}, t)=\mathscr{D}(t) \mathbf{x}+\frac{1}{2} \mathbf{w}(t) \times \mathbf{x}+\mathbf{A}(t) F(\boldsymbol{\alpha}(t) \cdot \mathbf{x}) \\
& =\left(\begin{array}{c}
\lambda\left(x_{1}-x_{2}\right) \\
\lambda\left(x_{1}-x_{2}\right) \\
0
\end{array}\right) \\
& +\left(\begin{array}{c}
0 \\
0 \\
\Gamma^{1 / 2}\left[c_{1} \cos (N t)-c_{2} \sin (N t)\right]
\end{array}\right) \\
& \cdot \sin \left(-x_{1}+x_{2}\right) \\
& \tilde{\rho}=\rho_{b}+\mathbf{b}(t) \cdot \mathbf{x}+B(t) F(\boldsymbol{\alpha}(t) \cdot \mathbf{x})=\rho_{b}+\rho(\mathbf{x}, t) \\
& =\rho_{b}-x_{3}+\left(c_{1} \sin (N t)+c_{2} \cos (N t)\right) \\
& \cdot \sin \left(-x_{1}+x_{2}\right) \\
& p=\frac{1}{2 \bar{P}} \widehat{P}(t) \mathbf{x} \cdot \mathbf{x}+P(t) G(\boldsymbol{\alpha}(t) \cdot \mathbf{x})=\frac{1}{2 \bar{P}} \\
& \left(\begin{array}{ccc}
\frac{\lambda}{R_{0}} & -\frac{\lambda}{R_{0}} & 0 \\
-\frac{\lambda}{R_{0}} & \frac{\lambda}{R_{0}} & 0 \\
0 & 0 & -\Gamma
\end{array}\right) \mathbf{x} \cdot \mathbf{x}+0 \\
& =\frac{1}{2 \bar{P}}\left[\frac{\lambda}{R_{0}}\left(x_{1}^{2}-2 x_{1} x_{2}+x_{2}^{2}\right)-\Gamma x_{3}^{2}\right] .
\end{aligned}
$$

Let us find the flow of fluids that concern Example 2. Consider that the fluid particle is at $\mathbf{x}(t)=\left(x_{1}, x_{2}, x_{3}\right)^{T}$ at any instant $t$. A particle is moving with velocity $\mathbf{v}(\mathbf{x}, t)$ and suppose that the particle is at position $\mathbf{x}(0)=\left(x_{10}, x_{20}, x_{30}\right)^{T}$ at time $t=0$. The flow of fluid is then defined through the $\operatorname{map} \beta: \mathbb{R} \times \mathbb{R}^{3} \mapsto \mathbb{R}^{3}$ as follows:

$$
\beta(t, \mathbf{x})=\mathbf{x}(t)=\left(\begin{array}{l}
x_{1}(t) \\
x_{2}(t) \\
x_{3}(t)
\end{array}\right)
$$

and this flow is completely determined by the following initial value problem for any $\mathbf{x} \in \mathbb{R}^{3}$ :

$$
\begin{aligned}
\frac{d \mathbf{x}}{d t} & =\mathbf{v}(\mathbf{x}, t), \\
\mathbf{x}(0) & =\left(x_{10}, x_{20}, x_{30}\right)^{T} .
\end{aligned}
$$




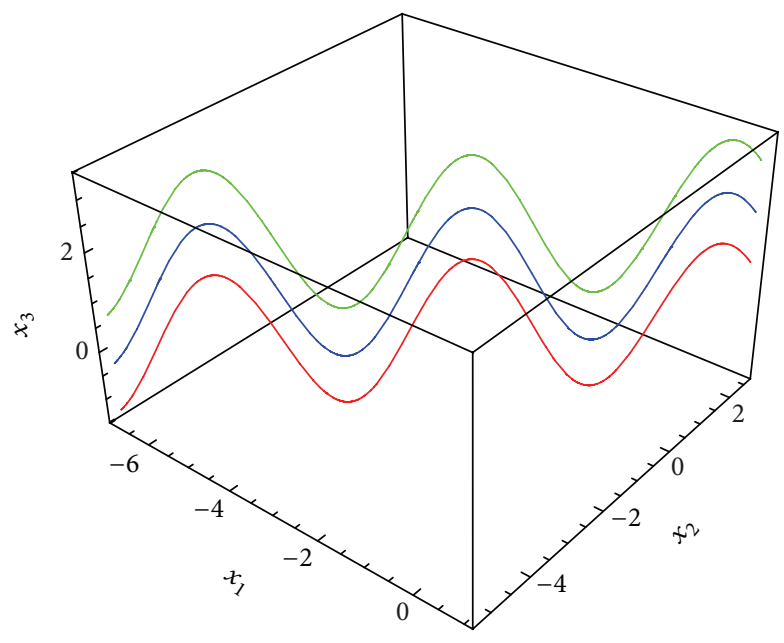

Figure 1: Integral curves.

In this example, we have the initial value problem as given below:

$$
\begin{aligned}
& \frac{d x_{1}}{d t}=\lambda\left(x_{1}-x_{2}\right) ; \quad x_{1}(0)=x_{10}, \\
& \frac{d x_{2}}{d t}=\lambda\left(x_{1}-x_{2}\right) ; \quad x_{2}(0)=x_{20}, \\
& \frac{d x_{3}}{d t}=\Gamma^{1 / 2}\left[c_{1} \cos (N t)-c_{2} \sin (N t)\right] \sin \left(-x_{1}+x_{2}\right) ; \\
& x_{3}(0)=x_{30} .
\end{aligned}
$$

We can solve the first and second equations of (47) explicitly and it is observed that $-x_{1}(t)+x_{2}(t)$ remain constant along the flow. Let us assume that $-x_{1}(t)+x_{2}(t)=L$ for all $t$. Hence, we have $x_{20}=L+x_{10}$. Also, we consider $c_{1}, c_{2}$ to be the particular constants. With these axioms a vector function given by right hand side of (47) is Lipschitz continuous in $\mathbf{x}$ over the vertical plane $-x_{1}+x_{2}=L$. Hence there exists a unique solution through each point on this plane. The integral curves passing through each of the points $\left(x_{10}, x_{20}, x_{30}\right)$ on this plane are given by

$$
\begin{aligned}
& x_{1}(t)=-\lambda L t+x_{10}, \\
& x_{2}(t)=-\lambda L t+L+x_{10} \\
& x_{3}(t)=x_{30}+\sin (L)\left[c_{1} \sin (N t)+c_{2} \cos (N t)-c_{2}\right] .
\end{aligned}
$$

To the given particular value of $L$, we have the particular integral curve passing through each of the points of the form $\left(x_{10}, x_{20}, x_{30}\right)$. In Figure 1, we have depicted these integral curves for $L=\pi / 2, \lambda=5, N=16$, and $c_{1}=c_{2}=1$ passing through the points $(1, \pi / 2+1,1),(1, \pi / 2+1,2)$, and $(1, \pi / 2+1,3)$ by red, blue, and green colored curves, respectively.

Furthermore, if we vary $L$ and initial points satisfying the condition $-x_{10}+x_{20}=L$, then (48) gives us a twoparameter family of integral surface. We have depicted this integral surface in Figure 2 for $-\pi / 2 \leq L \leq \pi / 2$.

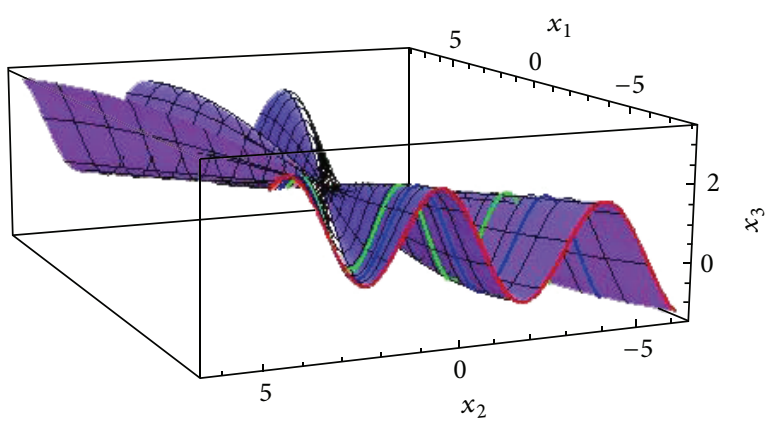

FIGURE 2: Integral surface.

\section{Conclusion}

In Section 2, we have the special exact solutions of rotating stratified Boussinesq equations (1) in the form of (9), whereas (12) gives us the nonlinear plane wave solutions. In Section 4, we develop the superimposed solutions of rotating stratified Boussinesq equations that are given by (21), which consists of addition of two components, one in the form of (9) and second component in the form of (12). We present these solutions in the form of Theorem 6. In support of these solutions, we have given two examples in Section 5.

\section{Competing Interests}

The authors declare that they have no competing interests.

\section{Acknowledgments}

The financial support to this research by University Grants Commission, New Delhi 110 002, India (Research Project F. no. 41-1380/2012 (SR)), is greatly acknowledged.

\section{References}

[1] A. E. Gill, Atmosphere-Ocean Dynamics, Academic Press, New York, NY, USA, 1982.

[2] K. R. Rajagopal, M. Ruzicka, and A. R. Srinivasa, "On the Oberbeck-Boussinesq approximation," Mathematical Models and Methods in Applied Sciences, vol. 6, no. 8, pp. 1157-1167, 1996.

[3] A. J. Majda and M. G. Shefter, "Elementary stratified flows with instability at large Richardson number," Journal of Fluid Mechanics, vol. 376, pp. 319-350, 1998.

[4] G. K. Srinivasan, V. D. Sharma, and B. S. Desale, "An integrable system of ODE reductions of the stratified Boussinesq equations," Computers \& Mathematics with Applications, vol. 53, no. 2, pp. 296-304, 2007.

[5] L. R. M. Maas, "Theory of basin scale dynamics of a stratified rotating fluid," Surveys in Geophysics, vol. 25, no. 3-4, pp. 249279, 2004.

[6] L. R. M. Maas, "A simple model for the three-dimensional, thermally and wind-driven ocean circulation," Tellus A, vol. 46, no. 5, pp. 671-680, 1994.

[7] A. M. Majda, Introduction to PDEs and Waves for the Atmosphere and Ocean, vol. 9 of Courant Lecture Notes in Mathematics, American Mathematical Society, Providence, RI, USA, 2003.

[8] B. S. Desale and V. Sharma, "Nonlinear plane waves in rotating stratified boussinesq equations," Nonlinear Dynamics and Systems Theory, vol. 13, no. 1, pp. 375-382, 2013. 


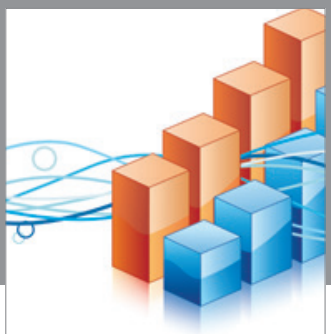

Advances in

Operations Research

vatem alat4

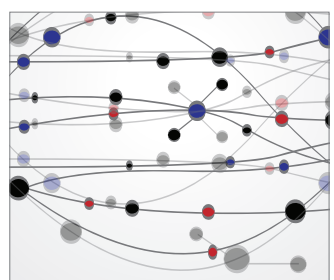

\section{The Scientific} World Journal
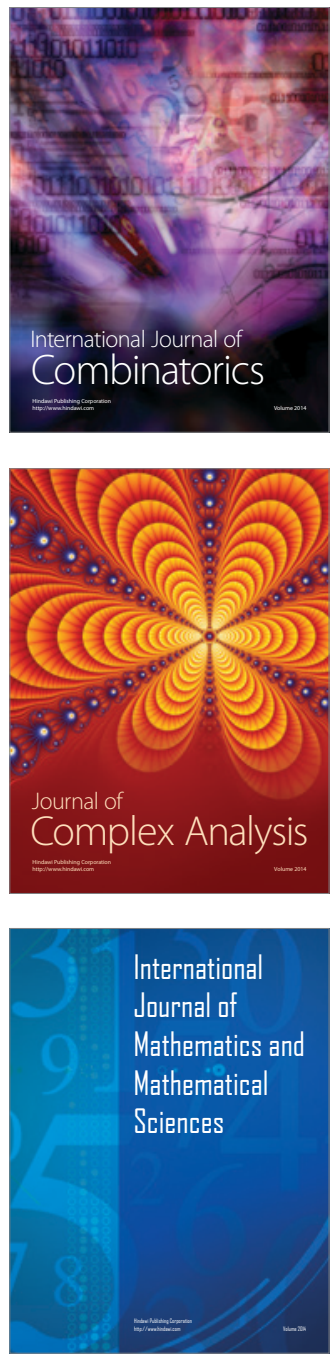
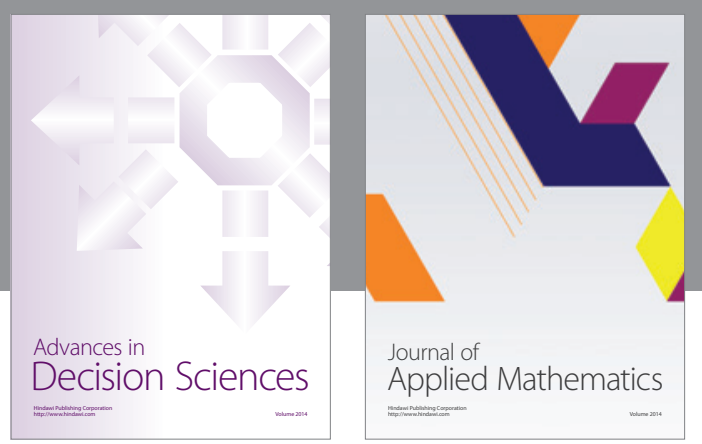

Algebra

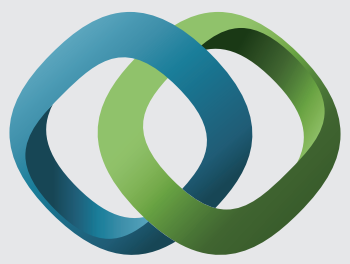

\section{Hindawi}

Submit your manuscripts at

http://www.hindawi.com
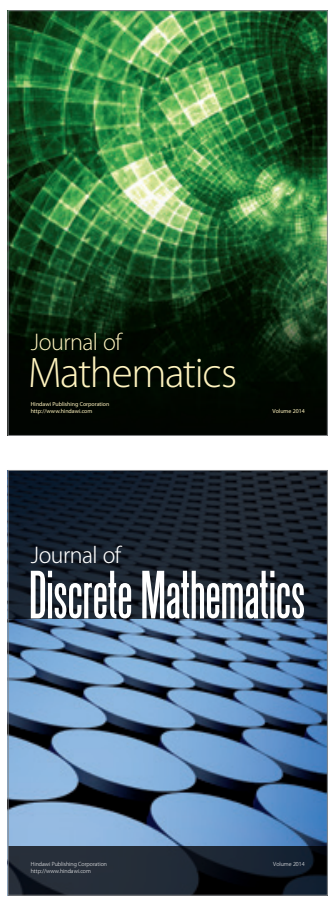

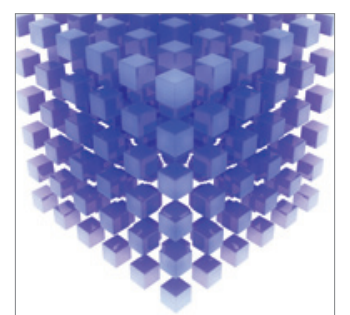

Mathematical Problems in Engineering
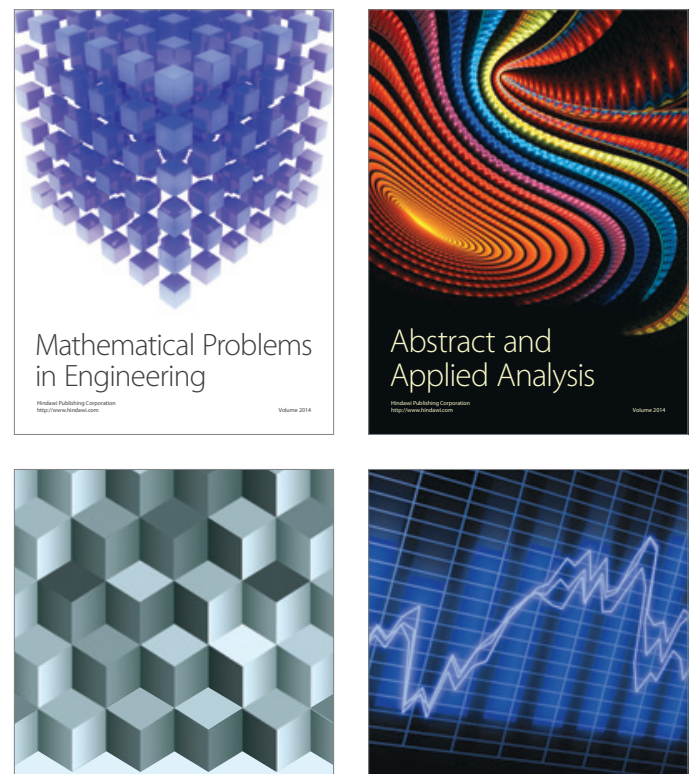

Journal of

Function Spaces

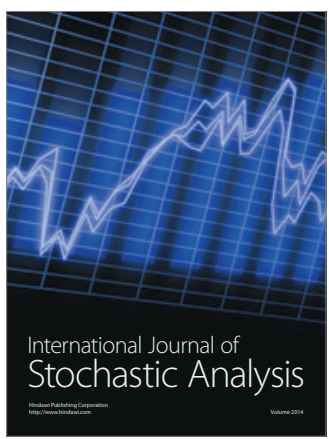

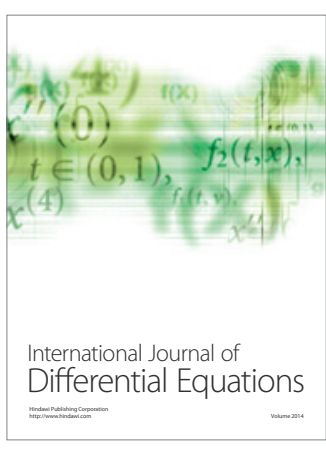
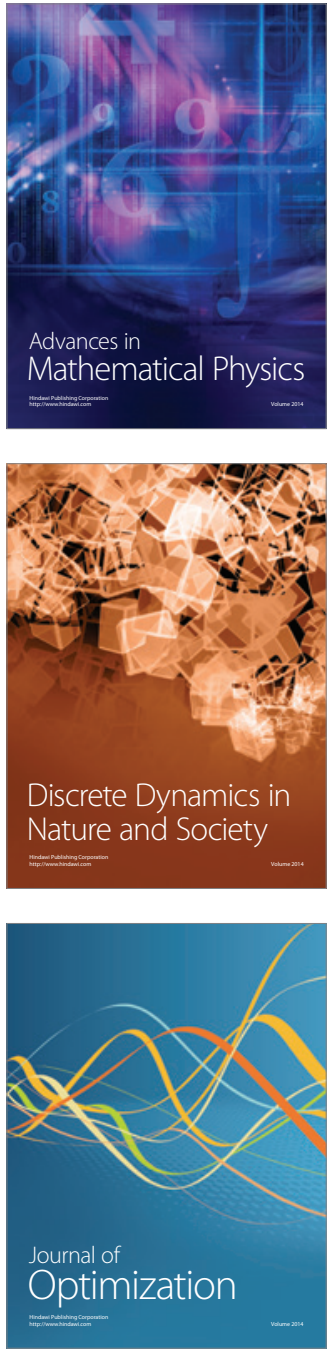